\title{
SNR and molecular cloud associations as seen by H.E.S.S.
}

\section{Cyril Trichard* on behalf of the H.E.S.S. collaboration}

Laboratoire d'Annecy-le-vieux de Physique des Particules

E-mail: cyril.trichardelapp.in2p3.fr

\begin{abstract}
Supernova remnants (SNRs) associated with molecular clouds are considered as very promising objects to point at the origin of Galactic cosmic rays (CRs). Molecular clouds act as very efficient target for collisions of accelerated particles, resulting in a subsequent enhancement of gammaray emission. Molecular clouds are therefore interesting objects to be observed by high-energy gamma-ray experiments. Several SNR and molecular cloud associations have been detected at high and very high gamma-ray energies and the hadronic signature of the high energy gamma-ray radiation has been observed from some of them. The detection by H.E.S.S. of several associations (W28, W51C, W49B and more recently G349.7+0.2) has shown that these sources have a softer spectra at $\mathrm{TeV}$ energies than their spectra at $\mathrm{GeV}$ energies, suggesting a spectral break or steepening with energy. Thus study of such associations appears of prime interest to understand the particle acceleration and diffusion. The latest results by H.E.S.S. will be presented and discussed in the light of their multi-wavelength context.
\end{abstract}

Cosmic Rays and the InterStellar Medium - CRISM 2014,

24-27 June 2014

Montpellier, France

${ }^{*}$ Speaker. 


\section{SNRs seen by H.E.S.S.}

The H.E.S.S. phase I system consists of an array of four Cherenkov telescopes detecting very high energy (VHE) $\gamma$-rays through their induced air shower in the energy domain between a few hundreds of $\mathrm{GeV}$ up to a few tens of TeV. With an angular resolution of the order of $0.1^{\circ}$, its field of view as large as $\sim 5^{\circ}$ together with its location in the southern hemisphere enables H.E.S.S. to deeply explore the Galactic plane, which has led to the detection of a high number of Galactic sources, and among them, supernova remnants (SNRs).

SNRs are assumed to accelerate particle to high energies $\left(\sim 10^{15} \mathrm{eV}\right)$ through diffusive shock acceleration [19]. One of the main H.E.S.S. results is the detection of $>100 \mathrm{TeV}$ particles accelerated by the SNR RX J1713.7-3946 [12]. However the origin of the $\gamma$-radiation is still debated. The Fermi-LAT collaboration published the $\mathrm{GeV}$ observations of this region [3] and when compared to the H.E.S.S. results, the most probable interpretation is that its non-thermal photon emission has leptonic origin. In this scenario the $\gamma$-rays are emitted in the wake of interactions between accelerated electrons and ambient photon fields via inverse-Compton processes. The $\gamma$-ray emission can also potentially be interpreted as of hadronic origin: accelerated cosmic protons interact with ambient matter localised in dense clouds and produce $\gamma$-rays by neutral pion decay [23]. The high density medium and an enhanced magnetic field would prevent the low energy hadrons from entering into the clouds; this can result into very hard GeV spectra. Such signatures would indirectly demonstrate that the SNR shocks are among the privileged sites of cosmic ray acceleration.

\section{The interest of $\mathrm{SNR} / \mathrm{MC}$ interactions}

Revealing clear evidence to distinguish between the two main $\gamma$-ray production mechanisms (leptonic and hadronic) is a tough task for the sample of detected SNRs at $\mathrm{GeV}$ and TeV energies. SNRs interacting with molecular clouds (MC) may help to solve the CR production origin question. The lifetime of high-mass stars is low and the supernovae of these progenitors occur in the vicinity of their parent molecular cloud. The expanding ejecta shock wave rapidly encounters and then propagates inside the dense medium. The high density of interstellar material should increase the rate of hadronic collisions between accelerated and confined particles at the shock wave (through fermi acceleration processes) and the cold matter. The $\gamma$-ray spectrum produced in these environmental conditions should differ from isolated SNR and the enhanced hadronic emission may be detected. The main feature is mostly visible at low energies by the spectral shape of the pion decay bump $(\sim 100 \mathrm{MeV})$. However the dense medium would age the shock wave faster than isolated ones. This would influence the acceleration efficiency of the SNR. The TeV $\gamma$-ray observations will inform us on the maximum energy that CRs can reach in these accelerators, and the CR diffusion from the acceleration site can be probed as well.

The very interesting spectral features of interacting SNRs were clearly revealed by the Fermi collaboration: the Fermi-LAT spectral analysis results of the sources W44 and IC443 have shown the presence of pion-decay-bump shape at lower energies [5]. This smoking gun evidence of the $\gamma$ ray hadronic origin from two SNRs is an important advance in the understanding of CR acceleration processes and sites. 


\section{H.E.S.S. results}

Observing the sub-class of SNR/MC associations in $\mathrm{GeV}$ and $\mathrm{TeV}$ is also a powerful tool. In particular at the $\mathrm{TeV}$ regime the H.E.S.S. collaboration has dedicated a good fraction of the observation time scrutinising many objects in the Galactic plane, including several SNRs.

\section{$3.1 \mathrm{~W} 28$}

W28 region holds a mixed-morphology supernova remnant located at $\sim 3-4 \mathrm{kpc}[25$ ]. The age of the expanding ejecta revealed by X-ray emission is estimated to be $\sim 36$ kyrs [33, 42]. Several molecular cloud complexes are spotted by ${ }^{12} \mathrm{CO}$ emission. The cloud located in the northern part of the SNR exhibits strong OH maser emissions indicating a physical interaction between the SNR and the clump.

$\mathrm{TeV} \gamma$-rays were detected by H.E.S.S. towards W28 [9]. Four hotspots of significant $\gamma$-ray excess were found to be coincident with MC positions. The southern MC complex shows a brighter emission than the northern one. This behaviour differs with the $\mathrm{GeV}$ emission. Indeed, a detailed study of Fermi-LAT data showed the brightest $\mathrm{GeV}$ emission is coincident with the northern cloud close to the position of the $\mathrm{OH}$ masers [26]. This opposite behaviour was first highlighted by the Agile satellite [24]. The diffusion of CRs from the SNR to the more distant molecular clouds could explain the $\gamma$-ray spectrum of each region. The latest measurement of CR ionisation rates deduced from chemical modeling in both molecular cloud complexes confirm the scenario of high energy CR diffusion [45].

Only the northern cloud (here after W28N) is physically interacting with the SNR, so the $\gamma$-ray spectrum of this source only is reported here. The Fermi-LAT spectrum is well fitted by a broken power law [1]. The energy at the break is $1.0 \pm 0.2 \mathrm{GeV}$. The index before the break is $2.09 \pm 0.08$ and $2.74 \pm 0.06$ after. The H.E.S.S. spectrum is in good agreement with the $\mathrm{GeV}$ range and shows a $\gamma$-ray spectral index of $2.66 \pm 0.27$.

\subsection{W49B}

W49B is a well known $\gamma$-ray emitting SNR [2]. It exhibits a strong interaction with molecular clouds through several tracers [34, 47]. The distance was first estimated to be $\sim 8 \mathrm{kpc}$ [41]. A later study suggests W49 could be at the same distance as W49A, the HII region in its neighborhood, at $\sim 11.4 \mathrm{kpc}$ [15]. The latest observations in radio and infrared wavelengths support the farthest estimation and conclude to a distance of $\sim 10 \mathrm{kpc}$ [47]. The age of this SNR is estimated to be $\sim 4$ kyr [32].

Detection of $\mathrm{TeV} \gamma$-rays from W49B was reported by the H.E.S.S. collaboration [17]. The position of the excess nicely matches the Fermi-LAT source. The spectrum evolution from few hundreds of $\mathrm{MeV}$ to $\sim 10 \mathrm{TeV}$ is smooth and shows a steepening at higher energies. The $\mathrm{GeV}$ band exhibits a broken power law ( $\left.E_{\text {break }}=4.8 \pm 1.6\right)$ with an index of $2.18 \pm 0.04$ before the break and $2.9 \pm 0.2$ after [2]. The H.E.S.S. spectrum is nicely compatible with Fermi-LAT data and well described by a power law with an index of $3.1 \pm 0.3$ [17]. 


\subsection{G349.7+0.2}

G349.7+0.2 is a young SNR with an age of $\sim 1800$ yrs. Its distance, first estimated at $\sim 22$ $\mathrm{kpc}$ [22], was revisited to $11 \mathrm{kpc}$ close to the far $3 \mathrm{kpc}$ arm [43]. The age of the remnant was accordingly re-evaluated. The proximity of a dense molecular cloud was brought out by ${ }^{12} \mathrm{CO}$ emission coincident with the radio shell [16, 20]. Five $\mathrm{OH}$ masers were detected [22] matching the position of the molecular cloud and the remnant. A strong thermal X-ray emission highly correlated with the radio continuum emission [36] shows two plasma components. One from the ejecta and one from the hot shocked cloud.

G349.7+0.2 is a $\gamma$-ray emitting SNR. A GeV radiation was detected by Fermi-LAT at the position of the remnant [18]. The GeV spectrum is well described by a power law up to 300 $\mathrm{GeV}$. A new analysis include the latest version P7Rep and a bigger set of Fermi-LAT data gives consistent results. A featureless spectral power law can be derived from the Fermi-LAT data with an index of $2.19 \pm 0.04$ from $200 \mathrm{MeV}$ to $300 \mathrm{GeV}$ [27]. H.E.S.S. reported the detection of a TeV source coincident with the radio shell [44, 31]. The TeV excess position remarkably matches the Fermi-LAT source and the $\mathrm{OH}$ maser locations. The $\mathrm{TeV}$ spectrum is fitted by a power law from $\sim 200 \mathrm{GeV}$ to tens of $\mathrm{TeV}$, and no hint of cutoff was found. The spectral index significantly differs from $\mathrm{GeV}$ range since the index is $2.8 \pm 0.3$. The H.E.S.S. results clearly show the existence of a break between $\mathrm{TeV}$ and $\mathrm{GeV}$ range but such feature occurs at higher energies than for W28 and W49B, above $10 \mathrm{GeV}$ [27].

\subsection{Other candidates}

The association of a $\gamma$-ray excess to a specific counterpart is generally a tough task. This is mainly due to the spatial proximity of several objects known as possible $\gamma$-ray emitters, specially in the Galactic plane, combined with the limited resolution of $\gamma$-ray instruments. Several possible SNR/MC associations seen by H.E.S.S. are in this situation. The $\gamma$-rays are likely emitted by hadronic interactions due to the dense material in which the shock wave propagates, however, other scenarios for the $\gamma$-ray emission such as a pulsar wind nebula (PWN) can not be ruled out and make the interpretation less straightforward.

W51 is a good example. H.E.S.S. observed this region and discovered a significant $\gamma$-ray excess at $\mathrm{TeV}$ energies [21]. The emission exhibits an extension and possibly both the PWN and the SNR/MC interaction are emitting $\gamma$-rays. The GeV photons observed by Fermi-LAT cannot help to distinguish both contributions [39, 6]. W51 is a very good candidate to study the TeV emission from the propagation of SNR shock wave inside a dense molecular cloud if each astrophysical contribution could be well determined.

CTB 37A is a SNR close to the very bright RX J1713.7-3946. As for the two candidates cited above, a TeV excess coincident with dense material and $\mathrm{OH}$ masers was observed by H.E.S.S. [10]. A GeV source detected by Fermi-LAT is coincident with the excess [39] observed by H.E.S.S.. The presence of an X-ray compact source, located in the same $\gamma$-ray emission region, can be an alternative explanation of the origin of the $\gamma$-ray flux.

The $\gamma$-ray spectra of W51 and CTB 37A are different compared to the previous mentioned sources. The Fermi-LAT data can be fitted by a LogParabola model [39, 6] while the H.E.S.S. spectrum is well described by a power law [10, 14]. 
W41 is similar to W51. $\mathrm{TeV}$ and $\mathrm{GeV} \gamma$-rays were detected from this region [39, 28]. The TeV excess shows two spatial components, a point like source and a surrounding extended emission. The overlapping of the $\gamma$-ray emission with two possible $\gamma$-ray emitters, first a X-ray CCO counterpart, possibly associated with a PWN, and second, a high density medium traced by ${ }^{13} \mathrm{CO}$ and coincident with $\mathrm{OH}$ masers makes both scenarios (PWN and SNR/MC) probable explanations for the high energy emission. The spectral fit quality of both models can not exclude any interpretation. The $\gamma$-ray spectrum consists of two different power laws at $\mathrm{GeV}$ and $\mathrm{TeV}$ band and a $\Delta \Gamma \sim 0.5$.

Another good candidate is HESS J1745-303. A bright TeV emission was observed since 2006 [7], and follow up observations allowed the investigation of hadronic emission from SNR/MC associations [11]. This region is composed by several SNRs and one of them $(G 359.1+0.5)$ holds $\mathrm{OH}$ masers that follow radio boundaries [46]. However the search for MWL counterparts was not successful to fully understand the extended $\mathrm{TeV}$ emission. Pion decay radiation from hadronic collisions still remains the most probable explanation. Given the complexity of the region under examination no $\mathrm{GeV}$ clear spectrum corresponding to H.E.S.S. observations was derived, so the results of this source cannot be reported here.

Finally, Puppis A is a unique case of SNR/MC association. Fermi-LAT detected this source [39, 6]. H.E.S.S. observations toward this association led to a non detection [29] and the upper limits are incompatible with Fermi-LAT spectrum extrapolation. This non detection suggests a spectral break or an exponential cutoff before the H.E.S.S. energy domain.

\subsection{SNRs in dense medium}

H.E.S.S. observed many candidates of SNR/MC interactions. The ones cited above show strong tracers of the propagation of the SNR shock wave inside the molecular clouds, such as $\mathrm{OH}$ masers or excited molecular lines. But H.E.S.S. also detected several SNR evolving in dense interstellar medium where no tracers of strong interaction were detected yet. Good examples are the two close sources HESS J1640-465 and HESS J1641-463. The first one is a very luminous $\mathrm{TeV} \gamma$-ray source [30]. The favored scenario of this emission is hadronic radiation because of good overlap between $\gamma$-ray extended emission and dense interstellar material. HESS J1641-463 is located in this neighborhood [Ð. Its position also matches the dense interstellar matter position.

Several SNRs show tracers of strong interaction with dense molecular clouds but the $\gamma$-ray emission origin is likely associated to a PWN. The determination of the hadronic emission from escaping CRs in such objects would be a very difficult task. Kes78 [35] and W30 [8] are in this situation.

\section{Summary}

Table 11 summarizes the Fermi-LAT and H.E.S.S. results on SNR/MC associations described in the previous sections. W 28N, W49B and G349.7+0.2 are $\gamma$-ray sources for which the interaction of the SNR and the MC is the production site of the $\gamma$-rays.

All sources exhibit a similar and complex spectral shape. None of the $\gamma$-ray spectra can be described by a featureless power law from hundreds of $\mathrm{MeV}$ to tens of $\mathrm{TeV}$ and a broken power law appears to be the best model for most of the cases. The spectral index before the break is quite hard $\gtrsim 2.2$. The position of the spectral break may vary in at least one (and maybe two) 
Table 1: Summary of the $\gamma$-ray spectra at $\mathrm{GeV}$ and $\mathrm{TeV}$ energies seen by Fermi-LAT and H.E.S.S. respectively, for the sources described in section 3. Only the sources with a certain SNR/MC association are reported here. The four first sources of $\gamma$-ray emission originate from the interaction between the SNR and the dense clouds (Puppis A is separated since only U.L. were derived from H.E.S.S. data). The three sources listed below are separated because of possible contamination of the detected $\gamma$-rays by other sources in their neighborhood. W44 and IC443, which exhibit clear hadronic spectral signatures, are shown for comparison.

\begin{tabular}{|ccccccc|}
\hline Source & $\begin{array}{c}\text { Age } \\
(\mathrm{kyr})\end{array}$ & $\begin{array}{c}\text { Distance } \\
(\mathrm{kpc})\end{array}$ & $\begin{array}{c}E_{\text {break }} \\
(\mathrm{GeV})\end{array}$ & $\Gamma_{E<\text { break }}$ & $\Gamma_{E>\text { break }}$ & $\Gamma_{\mathrm{TeV}}$ \\
\hline \hline W28N & $\sim 36$ & $\sim 2$ & $1 \pm 0.2$ & $2.09 \pm 0.08$ & $2.74 \pm 0.06$ & $2.66 \pm 0.27$ \\
W49B & $\sim 4$ & $\sim 10$ & $\begin{array}{c}4.8 \pm 1.6 \\
\text { G349.7+0.2 }\end{array}$ & $2.18 \pm 0.04$ & $2.9 \pm 0.2$ & $3.1 \pm 0.3$ \\
& & $\sim 12$ & $>10$ & $2.19 \pm 0.04$ & - & $2.8 \pm 0.3$ \\
Puppis A & $\sim 4-8$ & $\sim 2$ & $?$ & $2.6 \pm 0.13$ & - & U.L. \\
& & & & & & \\
CTB 37A & - & $6-10$ & - & LogParabola & - & $2.3 \pm 0.13$ \\
W51 & $\sim 30$ & $\sim 6$ & - & LogParabola & - & detected \\
W41 & $\sim 60-200$ & $\sim 4$ & $?$ & $2.15 \pm 0.12$ & - & $2.64 \pm 0.13$ \\
& & & & & & - \\
W44 & $\sim 10$ & $\sim 3$ & $\sim 2$ & $2.36 \pm 0.05$ & $3.5 \pm 0.3$ & \\
IC443 & $\sim 10$ & $1-2$ & $\sim 20$ & $2.36 \pm 0.02$ & $3.1 \pm 0.1$ & $3.1 \pm 0.3$ Magic [13] \\
\hline
\end{tabular}

order of magnitude. The TeV spectra are very steep $\Gamma_{T e V} \gtrsim 2.7$, and no hint of cutoff at highest energies has been found. The determination of these spectral features is a key topic to understand the acceleration of CRs in SNRs.

The complementary use of Fermi-LAT and H.E.S.S. data provides very useful information to constrain the CR acceleration models. The observed deviation from the standard DSA theory [19], is an active field, and several explanations were proposed including Alfven wave damping [38] and diffusion of escaping particles [40, 37]. All models strongly depend on the environmental conditions and will not be discussed here.

The spectral steepening of G349.7+0.2 and Puppis A occurs above tens of GeV. With the new observation conducted with its $36 \mathrm{~m}$ dish fifth telescope, H.E.S.S. in its phase II, will be very efficient in this domain, where Fermi-LAT lacks statistics, and will better constrain the position of the break and the spectral steepening with the energy. The population size of SNR/MC associations seen at $\mathrm{GeV}$ and $\mathrm{TeV}$ is still low. To determine the universal $\mathrm{CR}$ acceleration parameters from SNRs a bigger amount of sources is required. The next generation Cherenkov system CTA is expected to contribute in a definitive way.

\section{References}

[1] A. A. Abdo et al. ApJ, 718:348-356, July 2010.

[2] A. A. Abdo et al. ApJ, 722:1303-1311, October 2010.

[3] A. A. Abdo et al. ApJ, 734:28, June 2011. 
[4] A. Abramowski et al. ApJ, 794:L1, October 2014.

[5] M. Ackermann et al. Science, 339:807-811, February 2013.

[6] M. Ackermann et al. ApJS, 209:34, December 2013.

[7] F. Aharonian et al. ApJ, 636:777-797, January 2006.

[8] F. Aharonian et al. ApJ, 636:777-797, January 2006.

[9] F. Aharonian et al. A\&A, 481:401-410, April 2008.

[10] F. Aharonian et al. A\&A, 490:685-693, November 2008.

[11] F. Aharonian et al. A\&A, 483:509-517, May 2008.

[12] F. Aharonian et al. A\&A, 531:C1, July 2011.

[13] J. Albert et al. ApJ, 664:L87-L90, August 2007.

[14] J. Aleksić et al. A\&A, 541:A13, May 2012.

[15] C. L. Brogan and T. H. Troland. ApJ, 550:799-816, April 2001.

[16] C. L. Brogan et al. ApJ, 537:875-890, July 2000.

[17] F. Brun for the H.E.S.S. Collaboration. ArXiv e-prints, April 2011.

[18] D. Castro and P. Slane. ApJ, 717:372-378, July 2010.

[19] L. O. Drury. Reports on Progress in Physics, 46:973-1027, August 1983.

[20] G. Dubner et al. A\&A, 426:201-212, October 2004.

[21] A. Fiasson for the H.E.S.S. Collaboration. ArXiv e-prints, 2009.

[22] D. A. Frail et al. AJ, 111:1651, April 1996.

[23] S. Gabici and F. A. Aharonian. MNRAS, 445:L70-L73, November 2014.

[24] A. Giuliani et al. $A \& A, 516: L 11$, June 2010.

[25] C. Goudis. Ap\&SS, 40:91-110, March 1976.

[26] Y. Hanabata et al. ApJ, 786:145, May 2014.

[27] H.E.S.S. Collaboration. sub. to $A \& A, 2014$.

[28] H.E.S.S. Collaboration. ArXiv e-prints, July 2014.

[29] H.E.S.S. Collaboration. sub. to $A \& A, 2014$.

[30] H.E.S.S. Collaboration. MNRAS, 441:3640-3642, July 2014.

[31] J. Holder. ArXiv e-prints, July 2014.

[32] U. Hwang, R. Petre, and J. P. Hughes. ApJ, 532:970-979, April 2000.

[33] V. M. Kaspi et al. ApJ, 409:L57-L60, June 1993.

[34] J. W. Keohane, W. T. Reach, J. Rho, and T. H. Jarrett. ApJ, 654:938-944, January 2007.

[35] K. Kosack. International Cosmic Ray Conference, 7:76, 2011.

[36] J. S. Lazendic et al. ApJ, 618:733-743, January 2005.

[37] H. Li and Y. Chen. MNRAS, 421:935-942, April 2012. 
[38] M. A. Malkov, P. H. Diamond, and R. Z. Sagdeev. Physics of Plasmas, 19(8):082901, August 2012.

[39] P. L. Nolan et al. ApJS, 199:31, April 2012.

[40] Y. Ohira, K. Murase, and R. Yamazaki. MNRAS, 410:1577-1582, January 2011.

[41] V. Radhakrishnan et al. ApJS, 24:49, January 1972.

[42] J. Rho and K. J. Borkowski. ApJ, 575:201-216, August 2002.

[43] W. W. Tian and D. A. Leahy. ApJ, 783:L2, March 2014.

[44] C. Trichard for the H.E.S.S. Collaboration. 33rd ICRC, Rio de Janeiro, GA-EX 756, 2013.

[45] S. Vaupré et al. $A \& A, 568: A 50$, August 2014.

[46] F. Yusef-Zadeh, K. I. Uchida, and D. Roberts. Science, 270:1801-1804, December 1995.

[47] H. Zhu, W. W. Tian, and P. Zuo. ApJ, 793:95, October 2014. 